\title{
The AURKA Gene rs2273535 Polymorphism Contributes to Breast Carcinoma Risk - Meta-analysis of Eleven Studies
}

\author{
Xu-Guang Guo ${ }^{1,2,3}$, Lei Zheng ${ }^{3}$, Wei-Bo Feng ${ }^{4,5}$, Yong Xia ${ }^{1,2 *}$
}

\begin{abstract}
The rs2273535 polymorphism in the AURKA gene had proven to be associated with breast carcinoma susceptibility. Nevertheless, the results of different studies remain contradictory. A meta-analysis covering 28,789 subjects from eleven different studies was here carried out in order to investigate the association in detail. The random effects model was used to analyze the pooled odds ratios (ORs) and their corresponding $95 \%$ confidence intervals (95\% CIs). A significant relationship between the rs2273535 polymorphism and breast tumors was found in an allelic genetic model (OR: 1.076, 95\% CI: 1.004-1.153, $p=0.040, P_{\text {teterogenity }}=0.002$ ). No significant association was detected in a homozygote model (OR: $1.186,95 \%$ CI: $\left.0.990-1.423, P=0.065, P_{\text {hetrogeneity }}=0.002\right)$, a heterozygote model (OR: 1.016,95\% CI: 0.959-1.076, $p=0.064, P_{\text {hetergeneity }}=0.000$ ), a dominant genetic model (OR: 1.147, 95\% CI: $\left.0.992-1.325, p=0.217, P_{\text {heterogeneity }}=0.294\right)$ and a recessive genetic model (OR: $1.093,95 \%$ CI: 0.878$\left.1.361, p=0.425, P_{\text {heterogeneity }}=0.707\right)$. A significant relationship between the $\mathbf{r s} 2273535$ polymorphism in the AURKA gene and breast tumor in Asian group was found in an allelic genetic model (OR: 1.124, 95\% CI: 1.003-1.29, $\left.p=0.044, P_{\text {heterogeneity }}=0.034\right)$, a homozygote model (OR: $1.229,95 \%$ CI: $\left.1.038-1.455, p=0.016, P_{\text {heterogeneity }}=0.266\right)$ and a recessive genetic model (OR: $1.227,95 \%$ CI: 1.001-1.504, $\left.p=0.049, P_{\text {heterogeneity }}=0.006\right)$. A significant association was thus observed between the rs2273535 polymorphism in the AURKA gene and breast cancer risk. Individuals with the rs2273535 polymorphism in the AURKA gene have a higher risk of breast cancer in Asian populations, but not in Caucasians.
\end{abstract}

Keywords: Breast carcinoma - STK-15 polymorphism - cancer risk - meta-analysis,

Asian Pac J Cancer Prev, 15 (16), 6709-6714

\section{Introduction}

Cancer is an important public health problem in the world. According to the report from American Cancer Society, breast cancer alone is expected to account for $29 \%$ $(232,670)$ of all new cancers among women in America (Siegel et al., 2013). Approximately 232,340 new cases of invasive breast cancer and 39,620 breast cancer deaths are expected to occur among US women in 2013 (DeSantis et al., 2014). Breast cancer incidence rates increased slightly among African American women. Among females, breast cancer ranks first in women aged 20 years to 59 years, and lung cancer causes the most cancer deaths in those aged 60 years and older (Siegel et al., 2014).

According to the report from the National Office for Cancer Prevention and Control of China in 2013, the estimated number of female breast cancer cases was about 208 thousand. And the overall crude incidence rate was 32.43 per 100,000 , accounting for $16.20 \%$ of all cancer cases in women, ranking first among all cancer incidences (Zeng et al., 2014). The incidence rates in the eastern area and Middle area were similar, but in western areas, the incidence rate of the disease was 23.47 per 100,000, lowest among all areas (Wu et al., 2014).

The primary risk factors for breast cancer are female sex and older age. Other potential risk factors are as follows: genetics, lack of childbearing or lack of breastfeeding, higher levels of certain hormones, certain dietary patterns, and obesity. Recent studies have indicated that exposure to light pollution is a risk factor for the development of breast cancer.

Some genetic susceptibility may have a minor role in most cases. Overall, however, genetics is considered to be the foremost cause of 5-10\% of all cases. In those with zero, one or two affected relatives, the risk of breast cancer before the age of 80 is $7.8 \%, 13.3 \%$ and $21.1 \%$ with a subsequent mortality from the disease of $2.3 \%, 4.2 \%$, and $7.6 \%$ respectively. Of those with a first degree relative

${ }^{1}$ Department of Clinical Laboratory Medicine, The Third Affiliated Hospital of Guangzhou Medical University, ${ }^{2}$ Department of Internal Medicine, The Third Clinical College of Guangzhou Medical University, ${ }^{3}$ Department of Clinical Laboratory Medicine, Nanfang Hospital of Southern Medical University, Guangzhou, ${ }^{4}$ Department of Clinical Laboratory Medicine, Affiliated Xixiang People's Hospital of Guangdong Medical College, ${ }^{5}$ Department of Clinical Laboratory Medicine, Shenzhen Second People's Hospital of Baoan District, Shenzhen, China*For correspondence: gysyxy@gmail.com 
with the disease, the risk of breast cancer between the age of 40 and 50 is double that of the general population. In less than $5 \%$ of cases, genetics plays a more important role by causing a hereditary breast-ovarian cancer syndrome. This includes those who carry the BRCA1 and BRCA2 gene mutation. These mutations account for up to $90 \%$ of the total genetic influence with a risk of breast cancer of $60-80 \%$ for those affected. Other significant mutations include: p53, PTEN, and STK11, CHEK2, ATM, BRIP1, PALB2 and CXCL12 (Xia et al., 2014). In 2012, researchers said that there are four genetically distinct types of breast cancer and that in each type, hallmark genetic changes lead to many cancers.

Recently, some studies have reported increasing breast cancer risks associated with single nucleotide polymorphisms (SNPs) of Aurora kinase A (AURKA) gene (rs2273535, rs8173). AURKA (also referenced as Aurora-A, STK15, Aurora 2 and BTAK) is a serine/ threonine kinase that plays a crucial part in regulation of mitotic chromosomal segregation and centrosome function. The AURKA gene is conserved in chimpanzee, Rhesus monkey, dog, cow, mouse, rat, chicken, zebrafish, fruit fly, mosquito, C.elegans, M.oryzae, A.thaliana, rice, and frog.

A functional polymorphism $91 \mathrm{~T} \rightarrow \mathrm{A}$ in exon 4 (rs2273535) in the AURKA gene has been identified which causes an amino acid substitution changing phenylalanine to isoleucine. The $91 \mathrm{~A}$ allele has been involved in increased risk of breast cancer, colorectal cancer, ovarian cancer and esophageal squamous cell carcinoma.

The association of STK15 polymorphism with breast cancer risk has been investigated in several studies, while the conclusion is still inconclusive. For example, data from Egan's study provide the first evidence that polymorphic variations in STK15 are a genetic susceptibility factor in breast cancer (Egan et al., 2004), but Tchatchou et al. found the rs2273535 polymorphism is neither associated with familial breast cancer risk in the German population nor was it associated with breast cancer (Tchatchou et al., 2007).

To ascertain the relationship between the rs2273535 polymorphism in the AURKA gene and breast cancer risk, we performed this meta-analysis by pooling all the eligible studies.

\section{Materials and Methods}

\section{Publication Search and Inclusion Criteria}

The following keywords were searched in PubMed database without a language limitation: "cancer of breast", "breast cancer", "breast carcinoma", "carcinoma of breast", "breast neoplasms", "breast neoplasms", "STK15”, “Aurora”, "BTAK”, "rs2273535”, "genetic variation" and "polymorphism". Additional relevant studies were also found in the indexed references of the retrieved literatures. The latest research was updated on June 3, 2014, with publication years ranging from 1997 to 2014 .

The studies were selected based on the following inclusion criteria: studies that evaluate rs2273535 gene polymorphism and breast cancer, studies that diagnosis

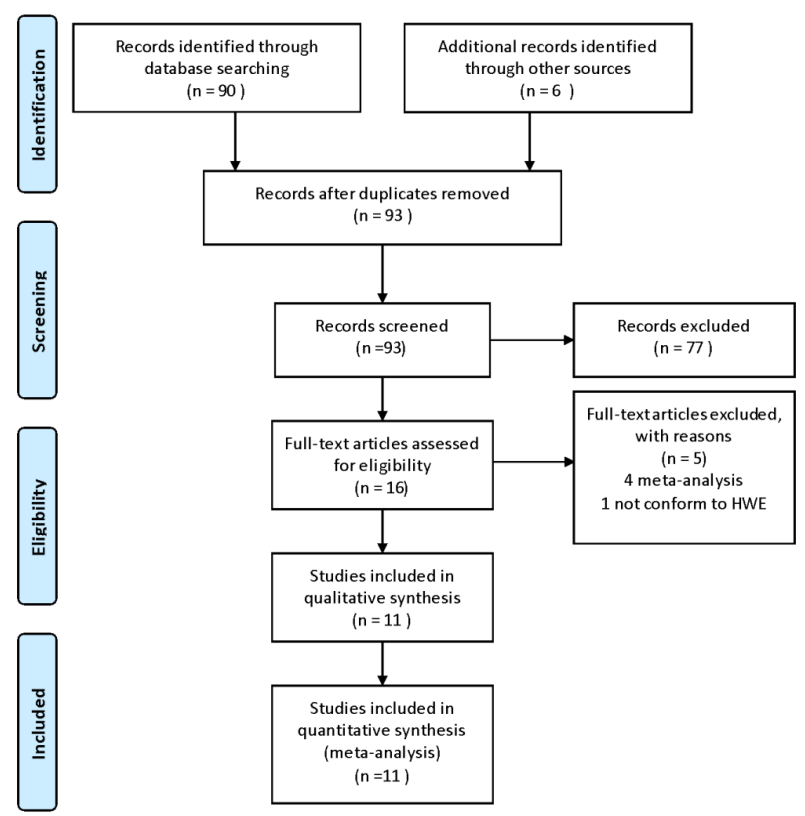

Figure 1. Flow Chart of the Study Selection Process

of cancer was confirmed by a histopathological analysis, case-control or cohort studies published in official journals, and studies that conform to the Hardy-Weinberg equilibrium (HWE). All records were selected by two authors independently according to the inclusion criteria and reached consensus on each record.

\section{Data extraction}

The data were abstracted according to a standard protocol. Studies that did not follow the inclusion criteria, those considered double publications, or those that provided inadequate data were excluded. If the same data appeared in different studies, the data were intended for use only once. The abstracted data comprised the following items: the first author's name, publication year, region, the number of genotypes, genotyping, study design, total number of cases and controls and HWE.

\section{Statistical analyses}

Five genetic models were used, including allelic (distribution of allelic frequency of the rs2273535 polymorphism in the AURKA gene, allelic model: A allele vs. T allele), homozygous (AA vs TT), heterozygous (AT vs TT) and recessive (AA vs AT+TT) and dominant (AA+AT vs TT) models (Thakkinstian et al., 2005). The odds ratios (ORs) and their corresponding $95 \%$ confidence intervals (CIs) were used to compare the association between the rs2273535 polymorphism in the AURKA gene and breast cancer. Chi-square-based Q-tests were used to calculate the heterogeneity between the individual studies with significance set at the $p<0.05$ level (Cochran, 1968). The random-effect model was used to assess the pooled OR (Der Simonian and Laird method) if there was heterogeneity among the individual studies (Mantel and Haenszel, 1959). Otherwise, the fixed-effect model was used (the Mantel Haenszel method). The pooled OR was determined through $\mathrm{Z}$ test with significance set at $p<0.05$ level.

Fisher's exact test was used to evaluate the HWE, and 
DOI:http://dx.doi.org/10.7314/APJCP.2014.15.16.6709

The rs2273535 Polymorphism in AURKA and Breast Carcinoma Risk

Table 1. Characteristics of Studies of The rs2273535 Polymorphism Included in this Pooled Analysis

\begin{tabular}{|c|c|c|c|c|c|c|c|c|c|c|c|c|c|}
\hline \multirow[t]{2}{*}{ Author } & \multirow[t]{2}{*}{ Year } & \multirow[t]{2}{*}{ Ethnicity } & \multicolumn{3}{|c|}{ Case } & \multicolumn{3}{|c|}{ Control } & \multirow[t]{2}{*}{ Genotyping } & \multirow{2}{*}{$\begin{array}{l}\text { Study } \\
\text { design }\end{array}$} & \multicolumn{2}{|c|}{ Sample size } & \multirow[t]{2}{*}{ HWE } \\
\hline & & & AA & AT & TT & AA & AT & $\mathrm{TT}$ & & & Case & Control & \\
\hline Shi & 2011 & Caucasian & 514 & 222 & 27 & 967 & 478 & 71 & Direct sequencing & Case-control & 763 & 1516 & Yes \\
\hline Ruan & 2011 & Asian & 167 & 568 & 599 & 161 & 691 & 716 & TaqMan & Case-control & 1334 & 1568 & Yes \\
\hline Consortiuma & 2010 & Caucasian & 107 & 1096 & 1873 & 249 & 1927 & 3290 & MALDI-TOF MS & Case-control & 3076 & 5466 & Yes \\
\hline Vidarsdottir & 2007 & Caucasian & 42 & 288 & 429 & 21 & 231 & 401 & TaqMan & Case-control & 759 & 653 & Yes \\
\hline Tchatchou & 2007 & Caucasian & 433 & 257 & 37 & 485 & 287 & 47 & TaqMan & Case-control & 727 & 819 & Yes \\
\hline Fletcher & 2006 & Caucasian & 18 & 154 & 335 & 48 & 280 & 547 & PCR-RFLP & Case-control & 507 & 875 & Yes \\
\hline Cox & 2006 & Caucasian & 66 & 401 & 774 & 65 & 571 & 1075 & TaqMan & Case-control & 1241 & 1711 & Yes \\
\hline Lo & 2005 & Asian & 348 & 288 & 71 & 886 & 887 & 196 & TaqMan & Case-control & 707 & 1969 & Yes \\
\hline Dai & 2004 & Asian & 490 & 491 & 121 & 534 & 503 & 149 & TaqMan & Case-control & 1102 & 1186 & Yes \\
\hline Sun & 2004 & Asian & 256 & 214 & 50 & 192 & 262 & 66 & PCR-RFLP & Case-control & 520 & 520 & Yes \\
\hline Egan & 2004 & Caucasian & 50 & 331 & 559 & 31 & 283 & 516 & Direct sequencing & Case-control & 940 & 830 & Yes \\
\hline
\end{tabular}

MALDI-TOF MS: Matrix-Assisted Laser Desorption/Ionization Time of Flight Mass Spectrometry; PCR-RFLP: polymerase chain reaction-restriction fragment length polymorphism; HWE: Hardy-Weinberg equilibrium

Table 2. Meta-Analysis of The Association between The rs2273535 Polymorphism in The AURKA Gene and Breast Cancer Risk

\begin{tabular}{|c|c|c|c|c|c|c|c|c|}
\hline \multirow[t]{2}{*}{ Polymorphism } & \multirow[t]{2}{*}{ Population } & \multirow{2}{*}{$\begin{array}{l}\text { Number } \\
\text { of studies }\end{array}$} & \multicolumn{3}{|c|}{ Test of association } & \multicolumn{3}{|c|}{ Test of heterogeneity } \\
\hline & & & OR & $95 \%$ CI & $P$ value & Model & $P$ value & $\mathrm{I}^{2}$ \\
\hline \multirow[t]{3}{*}{ A verse $\mathrm{T}$} & Caucasian & 7 & 1.047 & $0.959-1.145$ & 0.305 & REM & 0.013 & $62.80 \%$ \\
\hline & Asian & 4 & 1.124 & $1.003-1.29$ & 0.044 & REM & 0.034 & $65.50 \%$ \\
\hline & Overall & 11 & 1.076 & $1.004-1.153$ & 0.04 & REM & 0.002 & $64.50 \%$ \\
\hline \multirow[t]{3}{*}{ AA versus TT } & Caucasian & 7 & 1.151 & $0.853-1.551$ & 0.357 & REM & 0.001 & $72.80 \%$ \\
\hline & Asian & 4 & 1.229 & $1.038-1.455$ & 0.016 & REM & 0.266 & $24.30 \%$ \\
\hline & Overall & 11 & 1.186 & $0.990-1.423$ & 0.065 & REM & 0.002 & $64.50 \%$ \\
\hline \multirow[t]{3}{*}{ AT versus TT } & Caucasian & 7 & 1.016 & $0.951-1.086$ & 0.639 & REM & 0.667 & $0.00 \%$ \\
\hline & Asian & 4 & 1.015 & $0.902-1.141$ & 0.81 & REM & 0.492 & $0.00 \%$ \\
\hline & Overall & 11 & 1.016 & $0.959-1.076$ & 0.599 & REM & 0.774 & $0.00 \%$ \\
\hline \multirow[t]{3}{*}{$\mathrm{AA}$ versus $\mathrm{AT}+\mathrm{TT}$} & Caucasian & 7 & 1.091 & $0.878-1.354$ & 0.433 & REM & 0.002 & $71.30 \%$ \\
\hline & Asian & 4 & 1.227 & $1.001-1.504$ & 0.049 & REM & 0.006 & $75.90 \%$ \\
\hline & Overall & 11 & 1.147 & $0.992-1.325$ & 0.064 & REM & 0 & $72.00 \%$ \\
\hline \multirow[t]{3}{*}{$\mathrm{AA}+\mathrm{AT}$ versus $\mathrm{TT}$} & Caucasian & 7 & 1.036 & $0.947-1.132$ & 0.443 & REM & 0.19 & $31.10 \%$ \\
\hline & Asian & 4 & 1.073 & $0.960-1.200$ & 0.213 & REM & 0.482 & $0.00 \%$ \\
\hline & Overall & 11 & 1.042 & $0.976-1.113$ & 0.217 & REM & 0.294 & $15.80 \%$ \\
\hline
\end{tabular}

OR odds ratio; CI confidence interval; REM: random effects model

significance was set at the $p<0.05$ level. The funnel plot was used to estimate the potential publication bias (Stuck et al., 1998). Egger's linear regression test on the natural logarithm scale of the OR was used to assess the funnel plot asymmetry with significance set at the $p<0.05$ level (Egger et al., 1997). STATA 12.0 software was utilized for performing the statistical analyses (Stata Corp, College Station, TX, USA).

\section{Results}

\section{Characteristics of eligible studies}

Of the 96 articles that were initially identified in the search strategy, 80 studies were removed, including 3 duplicates, 77 studies during the title/abstract review, and 5 studies during the full-text review (Ewart-Toland et al., 2005; Couch et al., 2007; Qin et al., 2013; Tang et al., 2013; Xu et al., 2013) (Figure 1). Eleven studies satisfied all of the criteria and were included in this report (Dai et al., 2004; Egan et al., 2004; Sun et al., 2004; Lo et al., 2005; Cox et al., 2006; Fletcher et al., 2006; Tchatchou et al., 2007; Vidarsdottir et al., 2007; 2010; Ruan et al., 2011; Shi et al., 2011). There were seven studies of Caucasians, and four Asians. The distribution of genotypes in the controls of all studies was in accord with HWE. The data were collected from 11676 breast cancer cases and 17113 controls in this meta-analysis (Table 1, Figure 1).

\section{Pooled analysis}

A significant relationship between the rs2273535 polymorphism in the AURKA gene and breast tumor was found in an allelic genetic model (OR: 1.076, 95\% CI: $\left.1.004-1.153, p=0.040, P_{\text {heterogeneity }}=0.002\right)$ as shown in Table 2.

No significant association was detected in a homozygote model (OR: 1.186, 95\% CI: 0.990-1.423, $\left.p=0.065, P_{\text {heterogeneity }}=0.002\right)$, a heterozygote model (OR: $1.016,95 \%$ CI: 0.959-1.076, $\left.p=0.064, P_{\text {heterogeneity }}=0.000\right)$, a dominant genetic model (OR: $1.147,95 \%$ CI: $0.992-$ $\left.1.325, p=0.217, P_{\text {heterogeneity }}=0.294\right)$ and a recessive genetic model (OR: $1.093,95 \%$ CI: $0.878-1.361, p=0.425$, $P_{\text {heterogeneity }}=0.707$ ) as shown in Table 2 .

In the Caucasian subgroup analysis, no significant association was detected in an allelic genetic model (OR: $1.047,95 \%$ CI: 0.959-1.145, $\left.p=0.305, P_{\text {heterogeneity }}=0.013\right)$, homozygote model (OR: 1.151, 95\% CI: 0.853-1.551, $\left.p=0.357, P_{\text {heterogeneity }}=0.001\right)$, a heterozygote model (OR: $1.016,95 \%$ CI: $\left.0.951-1.086, p=0.639, P_{\text {heterogeneity }}=0.667\right)$, a dominant genetic model (OR: $1.036,95 \%$ CI: $0.947-$ $\left.1.132, p=0.443, P_{\text {heterogeneity }}=0.190\right)$ and a recessive genetic 


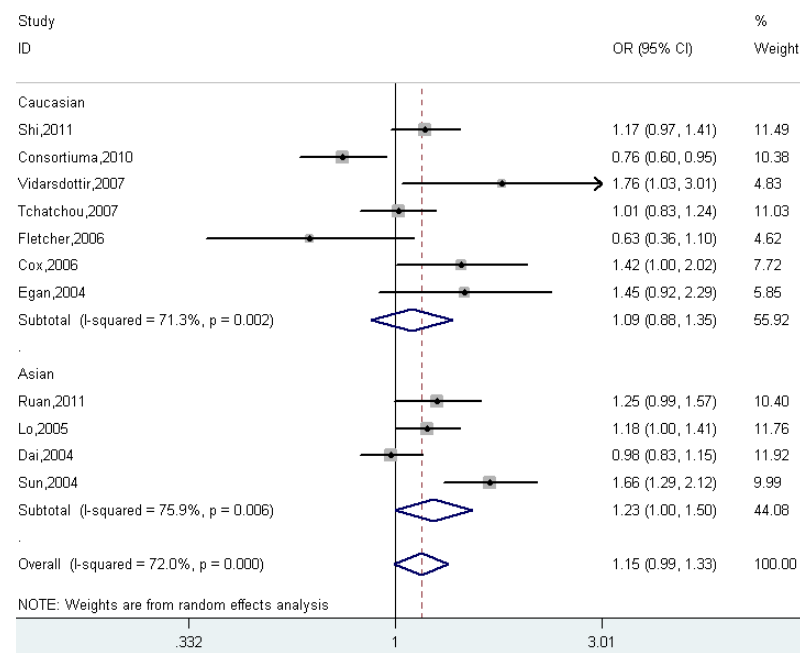

Figure 2. Forest Plot of Breast Cancer Associated with The rs2273535 Polymorphism in The AURKA Gene under A Recessive Genetic Model

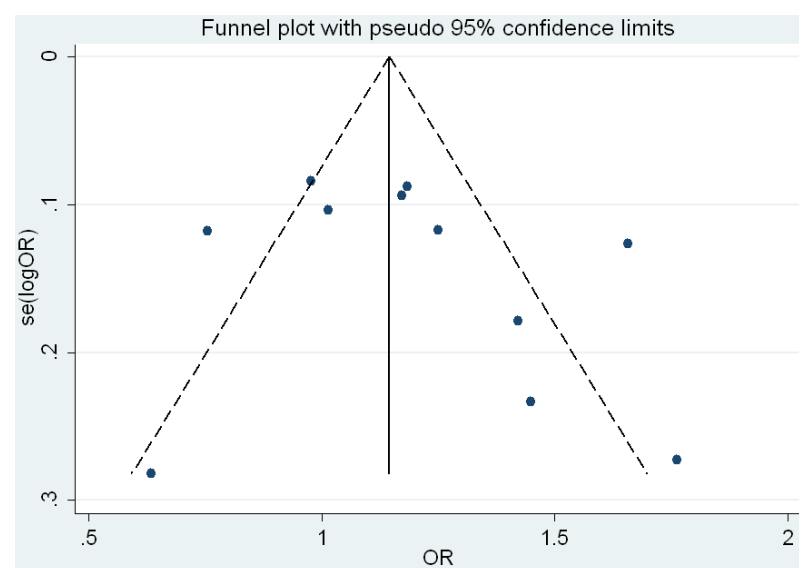

Figure 3. Funnel Plot for Studies of The Association of Breast Cancer and The rs2273535 Polymorphism in The AURKA Gene

model (OR: 1.091, 95\% CI: 0.878-1.354, $p=0.433$, $P_{\text {heterogeneity }}=0.002$ ) as shown in Table 2 .

A significant relationship between the rs2273535 polymorphism in the AURKA gene and breast tumor in Asian group was found in an allelic genetic model (OR: $1.124,95 \%$ CI: $\left.1.003-1.29, p=0.044, P_{\text {heterogeneit }}=0.034\right)$, a homozygote model (OR: 1.229, 95\% CI: 1.038-1.455, $\left.p=0.016, P_{\text {heterogeneity }}=0.266\right)$ and a recessive genetic model (OR: 1.227, 95\% CI: 1.001-1.504, $p=0.049$, $\left.P_{\text {heterogeneity }}=0.006\right)$ as shown in Table 2 .

No significant association was detected in Asian group in a heterozygote model (OR: $1.015,95 \% \mathrm{CI}$ : $\left.0.902-1.141, p=0.810, P_{\text {heterogeneity }}=0.492\right)$ and a dominant genetic model (OR: 1.073, 95\% CI: 0.960-1.200, $p=0.213$, $P_{\text {heterogeneity }}=0.482$ ) as shown in Table 2 and Figure 2 .

\section{Bias diagnosis}

The publication bias of the studies was evaluated using the funnel plot and Egger's test. Publication bias was not seen in the funnel plot (Figure 3). No statistically significant difference was discovered in the Egger's test $(p=0.164)$, indicating low publication bias in the current meta-analysis (Figure 4).

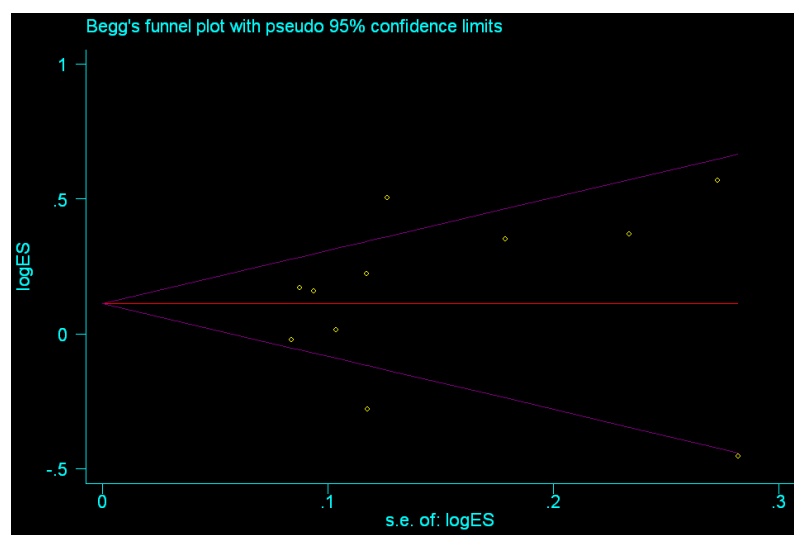

Figure 4. Begger's Funnel Plot about Studies of The Association of Breast Cancer a The rs2273535 Polymorphism in The AURKA Gene

\section{Discussion}

A significant relationship between the rs2273535 polymorphism in the AURKA gene and breast tumor was found in an allelic genetic model (OR: 1.076, 95\% CI: $\left.1.004-1.153, p=0.040, P_{\text {heterogeneit }}=0.002\right)$. A significant relationship between the rs2273535 polymorphism in the AURKA gene and breast tumor in Asian group was found in an allelic genetic model (OR: 1.124, 95\% CI: $\left.1.003-1.29, p=0.044, P_{\text {heterogeneity }}=0.034\right)$, a homozygote model (OR: 1.229, 95\% CI: $1.038-1.455, \mathrm{P}=0.016$, $\left.P_{\text {heterogeneity }}=0.266\right)$ and a recessive genetic model (OR: $1.227,95 \%$ CI: $1.001-1.504, p=0.049, P_{\text {heterogeneity }}=0.006$ ) as shown in Table 2.

The present study suggested a significant association was observed between the rs2273535 polymorphism in the AURKA gene and breast cancer risk. Individuals with the rs2273535 polymorphism in the AURKA gene have a higher risk of breast cancer in Asian populations, but not in Caucasians. This result was the strength of this meta-analysis.

Cancer is a disease in which abnormal cells grow in an uncontrolled way. Breast cancer is a cancerous growth that begins in the tissues of the breast. Breast cancer is the most common cancer in women, but it can also appear in men. In the US, it affects one in eight women.

There is information about factors that we know increase the risk, including cancer genes, as well as information about the factors that possibly increase the risk (Guo and Xia, 2013). Like most cancers, the risk of developing breast cancer increases as women get older. Having a mother or sister diagnosed with breast cancer approximately doubles the risk of breast cancer. But more than 8 out of 10 women who have a close relative with breast cancer will never develop it. If you have a very strong family history, there may be a faulty gene in your family that increases your risk of breast cancer. There are probably several gene faults that can increase breast cancer risk. We can test for them. The female sex hormone, oestrogen, and the male hormone, testosterone, can affect the development of breast cancer. In 2003, researchers from Cancer Research UK looked at hormone replacement therapy (HRT) and the risk of breast cancer in more than a million women. They showed clearly that HRT increases 
the risk of breast cancer while women take it and for up to 5 years afterwards. Other studies have also confirmed this. The million women study also showed that combined HRT (oestrogen and progesterone) is more likely to cause breast cancer than oestrogen only HRT. Other risk factor including the contraceptive pill, having children or having them early in life, When you start and stop having periods, Ethnic group, A previous breast cancer, ductal carcinoma in situ or lobular carcinoma in situ , Benign breast disease, Having dense breast tissue, Alcohol intake and smoking, Your weight and height, Chest X-rays or radiotherapy, Diabetes, Medicines, Shift work, and so on.

Commonly occurring single nucleotide polymorphisms (SNPs) have been shown to incrementally contribute to breast cancer risk. Although the effect of an individual SNP is generally small, the genetic effect of combinations of functionally relevant SNPs may additively or synergistically contribute to increased breast cancer risk. It is reported that a centrosomal serine/threonine kinase, serine/threonine kinase 15/breast tumor amplified kinase (STK15/BTAK, approved gene symbols are aurora2, ARK1, AIK1), has been identified as an oncogene. Some studies have shown that STK15/BTAK mRNA is over-expressed in a majority of breast cancers compared with normal breast tissues, and its over-expression is significantly associated with chromosomal instability, implicating STK15/BTAK in carcinogenesis through induction of chromosomal instability, though the possibility that STK15/BTAK over-expression is a simple consequence of chromosomal instability rather than a cause cannot be excluded (Miyoshi et al., 2001). Here we will discuss the rs 2273535 polymorphism in the AURKA gene and breast carcinoma risk.

Overall, a significant association exists between the rs2273535 polymorphism in the AURKA gene and breast carcinoma risk. This finding indicates that the genetic variant in AURKA gene exon3 may crucially modify the susceptibility of cancers (Yang et al., 2013).

Meta-analysis is a retrospective research that is subject to the methodological deficiencies of the included studies and several specific details merit consideration in the current meta-analysis. A first consideration is that our results are based on unadjusted estimates and a more precise analysis stratified by different lifestyle related habits and different grades of breast cancer could be performed if individual data were available. A second consideration is that large-scale studies on the relationship between the rs 2273535 polymorphism in the AURKA gene and breast cancer risk are still inadequate. AURKA is affected not only by gene polymorphism, but also by environmental factors. A third consideration is that Second, all recruited case-control studies were from Asians and Caucasians; thus, our results may only be suitable for these populations. Considering the complexity of cancer etiology and the low penetrance cancer susceptibility gene effects from STK15 F31I SNP, these important environmental factors should not be ignored.

Nevertheless, the total number of subjects contained in the present part of the analysis comprises the largest sample size so far. Finally, as with any meta-analysis of published results, the quality of our meta-analysis depends on that of the individual studies. Ideally we would like to pool individual level data. However, this is not possible in the present study. These considerations may distort our results.

Statistical surveys in America and England have shown that white women have a higher risk of breast cancer than women from other ethnic groups. This is at least partly due to lifestyle factors. To our surprise, the Ile/Ile homozygote frequency (rs2273535) in the Asian group in an allelic genetic model (OR: 1.124, 95\% CI: 1.003-1.29, $\left.p=0.044, P_{\text {heterogeneity }}=0.034\right)$ is much higher than in the control subjects, and also much higher than in Caucasian women in an allelic genetic model (OR: 1.047, 95\% CI: $\left.0.959-1.145, p=0.305, P_{\text {heterogeneity }}=0.013\right)$, indicating that individuals with the rs 2273535 polymorphism in the AURKA gene have a higher risk of breast cancer in Asian populations, but not in Caucasians.

To conclude, our meta-analysis demonstrated an association between the rs2273535 polymorphism in the AURKA gene and breast cancer risk. Nevertheless, large-scale and well-designed studies are required to investigate gene-gene and gene-environment interactions on the rs2273535 polymorphism in the AURKA gene and breast cancer risk, which may eventually lead to better comprehensive understanding of the possible roles in tumorigenesis.

\section{Acknowledgements}

This study was supported by grants from the Guangzhou Traditional Chinese Medicine and Western Medicine combined with Science and Technology Projects (No. 20122A011033).

\section{References}

Cochran WG (1968). The effectiveness of adjustment by subclassification in removing bias in observational studies. Biometrics, 24, 295-313.

Couch FJ, Sinilnikova O, Vierkant RA, et al (2007). AURKA F31I polymorphism and breast cancer risk in BRCA1 and BRCA2 mutation carriers: a consortium of investigators of modifiers of BRCA1/2 study. Cancer Epidemiol Biomarkers Prev, 16, 1416-21.

Cox DG, Hankinson SE, Hunter DJ (2006). Polymorphisms of the AURKA (STK15/Aurora Kinase) Gene and Breast Cancer Risk (United States). Cancer Causes Control, 17, 81-3.

Dai Q, Cai QY, Shu XO, et al (2004). Synergistic effects of STK15 gene polymorphisms and endogenous estrogen exposure in the risk of breast cancer. Cancer Epidemiol Biomarkers Prev, 13, 2065-70.

DeSantis C, Ma J, Bryan L, et al (2014). Breast cancer statistics, 2013. CA Cancer J Clin, 64, 52-62.

Egan KM, Newcomb PA, Ambrosone CB, et al (2004). STK15 polymorphism and breast cancer risk in a population-based study. Carcinogenesis, 25, 2149-53.

Egger M, Davey Smith G, Schneider M, et al (1997). Bias in meta-analysis detected by a simple, graphical test. $B M J$, 315, 629-34.

Ewart-Toland A, Dai Q, Gao YT, et al (2005). Aurora-A/STK15 $\mathrm{T}+91 \mathrm{~A}$ is a general low penetrance cancer susceptibility gene: a meta-analysis of multiple cancer types. Carcinogenesis, 26, 1368-73. 


\section{Xu-Guang Guo et al}

Fletcher O, Johnson N, Palles C, et al (2006). Inconsistent association between the STK15 F31I genetic polymorphism and breast cancer risk. J Natl Cancer Inst, 98, 1014-8.

Guo XG, Xia Y (2013). The Interleukin-18 promoter -607C >A polymorphism contributes to nasopharyngeal carcinoma risk: evidence from a meta-analysis including 1,886 subjects. Asian Pac J Cancer Prev, 14, 7577-81.

Lo YL, Yu JC, Chen ST, et al (2005). Breast cancer risk associated with genotypic polymorphism of the mitosisregulating gene Aurora-A/STK15/BTAK. Int J Cancer, 115, 276-83.

Mantel N, Haenszel W (1959). Statistical aspects of the analysis of data from retrospective studies of disease. J Natl Cancer Inst, 22, 719-48.

MARIE-GENICA Consortium on Genetic Susceptibility for Menopausal Hormone Therapy Related Breast Cancer Risk (2010). Polymorphisms in the BRCA1 and ABCB1 genes modulate menopausal hormone therapy associated breast cancer risk in postmenopausal women. Breast Cancer Res Treat, 120, 727-36.

Miyoshi Y, Iwao K, Egawa C, et al (2001). Association of centrosomal kinase STK15/BTAK mRNA expression with chromosomal instability in human breast cancers. Int $J$ Cancer, 92, 370-3.

Qin K, Wu C, Wu X (2013). Two nonsynonymous polymorphisms (F31I and V57I) of the STK15 gene and breast cancer risk: a meta-analysis based on 5966 cases and 7609 controls. $J$ Int Med Res, 41, 956-63.

Ruan Y, Song AP, Wang H, et al (2011). Genetic polymorphisms in AURKA and BRCA1 are associated with breast cancer susceptibility in a Chinese Han population. J Pathol, 225, 535-43.

Shi H, Bevier M, Johansson R, et al (2011). Single nucleotide polymorphisms in the $20 \mathrm{q} 13$ amplicon genes in relation to breast cancer risk and clinical outcome. Breast Cancer Res Treat, 130, 905-16.

Siegel R, Ma J, Zou Z, et al (2014). Cancer statistics, 2014. CA Cancer J Clin, 64, 9-29.

Siegel R, Naishadham D, Jemal A (2013). Cancer statistics, 2013. CA Cancer J Clin, 63, 11-30.

Stuck AE, Rubenstein LZ, Wieland D (1998). Bias in metaanalysis detected by a simple, graphical test. Asymmetry detected in funnel plot was probably due to true heterogeneity. $B M J, 316,70-1$.

Sun T, Miao X, Wang J, et al (2004). Functional Phe31Ile polymorphism in Aurora A and risk of breast carcinoma. Carcinogenesis, 25, 2225-30.

Tang W, Qiu H, Ding H, et al (2013). Association between the STK15 F31I polymorphism and cancer susceptibility: a meta-analysis involving 43, 626 subjects. PLoS One, 8, e82790.

Tchatchou S, Wirtenberger M, Hemminki K, et al (2007). Aurora kinases A and B and familial breast cancer risk. Cancer Lett, 247, 266-72.

Thakkinstian A, McElduff P, D'Este C, et al (2005). A method for meta-analysis of molecular association studies. Stat Med, 24, 1291-306.

Vidarsdottir L, Bodvarsdottir SK, Hilmarsdottir H, et al (2007). Breast cancer risk associated with AURKA 91T $>$ A polymorphism in relation to BRCA mutations. Cancer Lett, 250, 206-12.

Wu LZ, Han RQ, Zhou JY, et al (2014). Incidence and mortality of female breast cancer in Jiangsu, China. Asian Pac J Cancer Prev, 15, 2727-32.

Xia Y, Guo XG, Ji TX (2014). The G801A polymorphism in the CXCL12 gene and risk of breast carcinoma: evidence from a meta-analysis including 2,931 subjects. Asian Pac
J Cancer Prev, 15, 2857-61.

Xu L, Zhou X, Jiang F, et al (2013). STK15 rs2273535 polymorphism and cancer risk: A meta-analysis of 74, 896 subjects. Cancer Epidemiol, 38, 111-17.

Yang YC, Chang TY, Chen TC, et al (2013). Genetic variants in interleukin-18 gene and risk for cervical squamous cell carcinoma. Human Immunology, 74, 882-7.

Zeng H, Zheng R, Zhang S, et al (2014). Female breast cancer statistics of 2010 in China: estimates based on data from 145 population-based cancer registries. J Thorac Dis, 6, 466-70. 